\title{
Human herpesvirus 8-unrelated primary effusion lymphoma-like lymphoma following tyrosine kinase inhibitor treatment for chronic myelogenous leukemia
}

\author{
Minoru Kojima, ${ }^{1,2,3)}$ Naoya Nakamura, ${ }^{4)}$ Jun Amaki, ${ }^{1,2)}$ Hiroki Numata, ${ }^{1,2)}$ \\ Masashi Miyaoka, ${ }^{4)}$ Tadashi Motoori, ${ }^{5)}$ Koshi Matsumoto ${ }^{6}$ and Kiyoshi Ando ${ }^{2)}$
}

\begin{abstract}
A 69-year-old man was diagnosed with chronic myelogenous leukemia (CML) and treated with dasatinib. After two years on dasatinib, the patient achieved complete molecular response, but dasatinib treatment was discontinued due to exacerbation of pleural effusion. Nilotinib and imatinib were started but stopped due to an increase in pleural effusion. Thoracentesis was performed and he was diagnosed with human herpesvirus 8-unrelated primary effusion lymphoma (PEL)-like lymphoma. Complex chromosomal abnormality, including $B C L 6$ rearrangement, was found on chromosome analysis. To the best of our knowledge, this is the first report of PEL-like lymphoma following tyrosine kinase inhibitor treatment for CML.
\end{abstract}

Keywords: primary effusion lymphoma-like lymphoma, chronic myelogenous leukemia, tyrosine kinase inhibitor, $B C R-A B L$, $B C L 6$

\section{INTRODUCTION}

Chronic myelogenous leukemia (CML) is a chronic myeloproliferative neoplasm that originates from multipotent hematopoietic stem cells and is associated with the $B C R-A B L$ fusion gene located on the Philadelphia $(\mathrm{Ph})$ chromosome. ${ }^{1}$ Based on historical data prior to any effective therapy, median survival times for CML ranged between 2 and 3 years. ${ }^{2}$ However, in the current era of tyrosine kinase inhibitor (TKI) therapy using imatinib, dasatinib, nilotinib, and bosutinib, the prognosis has been markedly improved. ${ }^{3}$ Currently, an important issue is the management of adverse events. Pleural effusion is one of the major problems. The TKIs have different patterns of side effects, and pleural effusion is more frequently observed with dasatinib compared with imatinib, nilotinib, and bosutinib. ${ }^{4-7}$

Primary effusion lymphoma (PEL) is a rare type of nonHodgkin lymphoma (NHL) confined to the body cavities, usually without a tumor mass. ${ }^{1}$ It is associated with infection of human herpesvirus 8 (HHV-8), and Epstein-Barr virus (EBV) coinfection is common, especially with a background of human immunodeficiency virus type-1 (HIV) infection. ${ }^{8}$ However, some cases of HHV-8-negative primary lymphomatous effusion have been reported and termed HHV-8unrelated PEL-like lymphoma.,
In this report, we present a rare case of PEL-like lymphoma that was found during pleural effusion treatment with TKIs for CML.

\section{CASE REPORT}

A 69-year-old Japanese male was hospitalized for cerebral infarction. Peripheral blood evaluation revealed a hemoglobin level of $12.2 \mathrm{~g} / \mathrm{dl}$, a platelet count of $98.6 \times 10^{4} /$ $\mu 1$, a white blood cell count of $26,800 / \mu 1$ with $86 \%$ neutrophils, $6 \%$ lymphocytes, $1 \%$ monocytes, $1 \%$ eosinophils, $2 \%$ basophils, $2 \%$ myelocytes, and $2 \%$ metamyelocytes. Hepatitis B, Hepatitis C, HIV, and HTLV-1 were negative. Hypercellular bone marrow with an increase of megakaryocytes was observed on the bone marrow test, and the myeloblast count was less than 5\% (Figure1). Chromosomal analysis was abnormal with 46, XY, $\mathrm{t}(9 ; 22)(\mathrm{q} 34 ; \mathrm{q} 11.2)$ [20/20]. The patient was diagnosed with CML in the chronic phase. Dasatinib was started at $100 \mathrm{mg}$ once daily. After a year of dasatinib treatment, the patient achieved major molecular response (MMR). However, he developed dyspnea and bilateral pleural effusion was observed on chest X-ray; therefore, diuretics were started. After two years on dasatinib, the patient achieved complete molecular response (CMR), but dasatinib treatment was discontinued due to exacerbation of

\footnotetext{
Received: April 17, 2017. Revised: July 25, 2017. Accepted: July 31, 2017.

${ }^{1)}$ Department of Hematology, Ebina General Hospital, ${ }^{2}$ Division of Hematology/Oncology, Department of Internal Medicine, Tokai University School of Medicine, ${ }^{3)}$ Department of Internal Medicine, Sangenjaya-daiichi Hospital, ${ }^{4}$ Department of Pathology, Tokai University School of Medicine, ${ }^{5}$ Sagamihara Soubudai Pathology Institute, ${ }^{6}$ Department of Pathology, Ebina General Hospital

Corresponding author: Minoru Kojima, MD, PhD, Department of Internal Medicine, Sangenjaya-daiichi Hospital, 1-22-8 Sangenjaya, Setagaya-ku, Tokyo, 154-0024, Japan. E-mail: mkojima2920@gmail.com
} 
pleural effusion resistant to diuretics and steroids. Nilotinib at $150 \mathrm{mg}$ twice daily was selected because the pleural effusion had decreased, but the pleural effusion increased again, and nilotinib was discontinued after two weeks. Imatinib at $400 \mathrm{mg}$ once daily was started after discontinuation of nilotinib, but was stopped after only ten days because pleural effusion increased again. Even after TKIs were stopped, the pleural effusion did not decrease and his dyspnea remained. Thus, thoracentesis was performed. IgM, bcl-2, cytoplasmic CD79a, and $\kappa$-chain were positive on flow cytometry, and molecular testing on the pleural effusion sample indicated a monoclonal immunoglobulin heavy chain gene rearrangement. Microscopically, in the cytocentrifuge preparation of the pleural effusion, cells composed of mid to large-sized pleomorphic nuclei with abundant cytoplasm, and

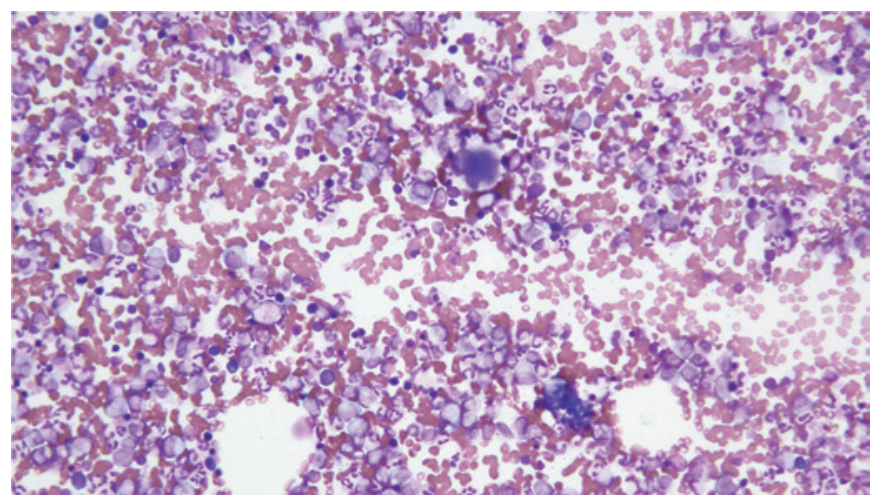

Fig. 1. Bone marrow test showed hypercellular bone marrow with an increase of megakaryocytes and a myeloblast count less than $5 \%$. perinuclear halo consistent with plasmacytoid differentiation were occasionally seen. Immunohistochemical staining for CD20, CD79a, CD99, BCL2, BCL6 and MUM-1 was positive, but MPO, CD3, CD5, CD7, CD10, CD34, CD56 and TdT were negative, and EBER RNA in situ hybridization was negative. The MIB-1 labeling index indicated more than 90\% tumor cells (Figure 2). HHV-8 immunohistochemistry was negative and HHV-8 DNA quantification in the pleural effusion was less than $100 \mathrm{copy} / \mathrm{mL}$. Chromosome analysis based on a G-banding analysis demonstrated the karyotype $82<3 \mathrm{n}>, \mathrm{XXY},+\mathrm{Y},+2,+\operatorname{der}(3) ? \mathrm{t}(3 ; 14)(\mathrm{q} 27 ; \mathrm{q} 32) \times 2, ? \mathrm{t}(3 ; 9 ; 14)$ (q2 7;p13; q32), -6,-7, add(7)(p22), +der(9)?t(3;9) $(\mathrm{q} 27 ; \mathrm{p} 13),+13,+\operatorname{add}(14)(\mathrm{q} 32),+18,+19,+21,+5$ mar. FISH analysis was negative for $B C R-A B L$ and positive for $B C L 6$ (Figure 3). The translocation of 9p13 may have caused an abnormality in $P A X-5$, but FISH analysis was not performed. Based on these findings, a diagnosis of PEL-like lymphoma was made. Mild hypocellular marrow without lymphoma cells and dysplasia was found on bone marrow biopsy, and the marrow karyotype was normal. The patient was initially treated for de novo PEL-like lymphoma with the R-CHOP (rituximab, cyclophosphamide, vincristine, doxorubicin, and predonisolone) regimen. After two cycles of R-CHOP, doxorubicin was stopped due to possible anthracycline cardiomyopathy even though the patient had no risk of cardiac disease before R-CHOP. After 3 additional cycles of R-CVP treatment, we stopped the chemotherapy because of systemic edema due to heart failure. Computed tomography (CT) revealed remnant bilateral pleural effusion, but FDG accumulation was not observed with Positron Emission Tomography

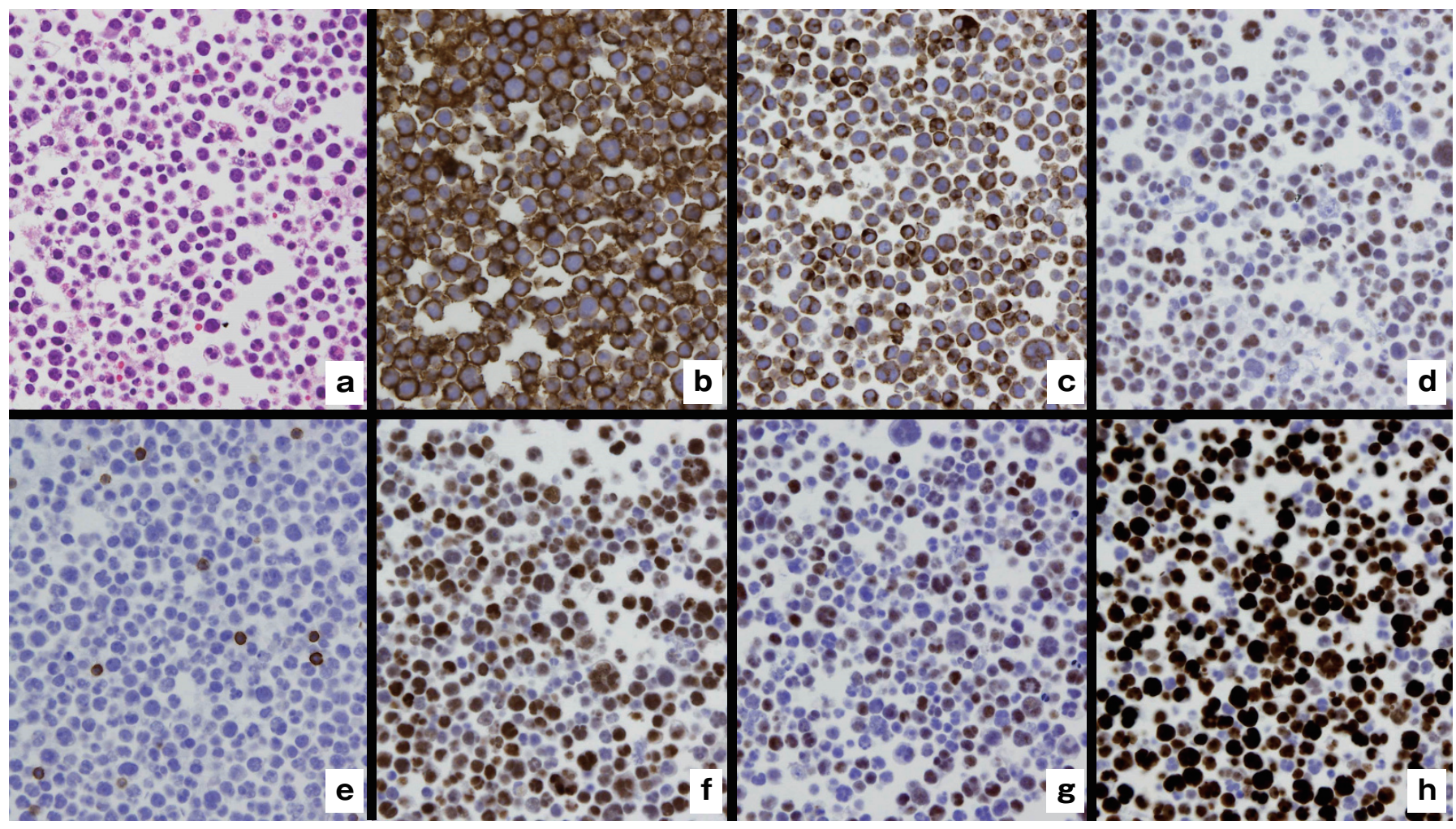

Fig. 2. Histopathological and immunohistochemical appearance of PEL-like lymphoma. (a) Hematoxylin-eosin staining, (b) CD20, (c) BCL2, (d) BCL6, (e) CD3, (f) CMYC, (g) MUM1 and (h) MIB1 
(PET)-CT. As lymphoma cells were not observed on thoracentesis, he was considered to have achieved complete remission. After chemotherapy for PEL-like lymphoma, $B C R$ $A B L \mathrm{IS} \%$ increased (0.6795) and he was diagnosed with loss of MMR. The patient began bosutinib treatment, but discontinued due to diarrhea and anorexia. Nilotinib was re-started and the patient achieved MMR.

\section{DISCUSSION}

CML is thought to arise from a multi-potent hematopoietic stem cell that has undergone BCR-ABL translocation, resulting in a shortened chromosome 22 , referred to as the Philadelphia $(\mathrm{Ph})$ chromosome. Takahashi et al. reported that the Ph chromosome was found in pluripotent stem cells, but in a lower incidence among B lymphocytes than among neutrophils and monocytes. ${ }^{11}$ Their hypothesis is that lymphocytes with the $\mathrm{Ph}$ chromosome fail to differentiate and the usual target of malignant transformation in CML is a more restricted stem cell committed to B-cell lineages. This is consistent with the B-cell population being chimeric with respect to $B C R-A B L$. In our case, $B C L 6$ was positive, but $B C R-A B L$ was negative in FISH analysis; therefore, PEL like lymphoma originated from $B C L-A B L$-negative B lymphocytes. Non-Hodgkin lymphoma during CML treatment is rare. Ichinohasama $\mathrm{R}$ et al. reported two cases of $\mathrm{Ph}$-negative non-Hodgkin lymphoma occurring in the chronic phase of Ph-positive CML, defined as a genetically different neoplasm from extramedullary localized blast crisis. ${ }^{12}$ The author advocates there are three types of lymphoid neoplasms related with the $\mathrm{Ph}$ chromosome or status of CML as follows: Type A: Lymphoid blast crisis (both localized or not localized) consisting of Ph-positive cells in CML (same genetic background for both lymphoid neoplasm and CML), Type B: Ph-negative lymphoid neoplasm occurring in CML (different genetic background between lymphoid neoplasm and CML), and Type $\mathrm{C}$ : Ph-positive lymphoid neoplasm in a patient without $\mathrm{Ph}$-positive disorders. It is very important to distinguish the subtypes to determine the appropriate treatment. Our case corresponds with Type B, and he was in complete remission after rituximab-combined chemotherapy, which is the standard treatment for B-cell lymphoma, without needing a stronger regimen.

In long-term follow-up of imatinib treatment for CML, the incidence of secondary neoplasm was $12 / 551(2.2 \%)$ and that of hematological neoplasm was $5 / 551(0.9 \%){ }^{13}$ Thus, the incidence of secondary neoplasm was not increased by imatinib treatment for CML. Dasatinib treatment is also similar. ${ }^{14}$ The onset mechanism of neoplasms by TKI is unclear. Kawamata et al. reported that imatinib reduces expression of AID by off-target effects, and it suppresses class switching and causes low gammaglobulinemia. ${ }^{15}$ It is unknown whether dasatinib has the same effects, but if we apply this hypothesis to our case, lymphoma may have developed due to hypo-gammaglobulinemia. On the other hand, pleural effusion associated with TKIs is more frequently observed with dasatinib than with other TKIs. ${ }^{16}$ The mechanism by which dasatinib induces pleural effusion is likely related to off-target kinase inhibition. In addition to Abl, dasatinib potently inhibits platelet-derived growth factor receptor beta (PDGFRB), ${ }^{17,18}$ a receptor expressed in pericytes $^{19}$ that is involved in the regulation of angiogenesis. ${ }^{20}$ PDGFRB-deficient mouse embryos lack microvascular pericytes, and develop defective blood vessels and edema. ${ }^{21}$ Blockade of PDGFRB with CDP860, a humanized pegylated diFab in patients with advanced ovarian and colorectal
A

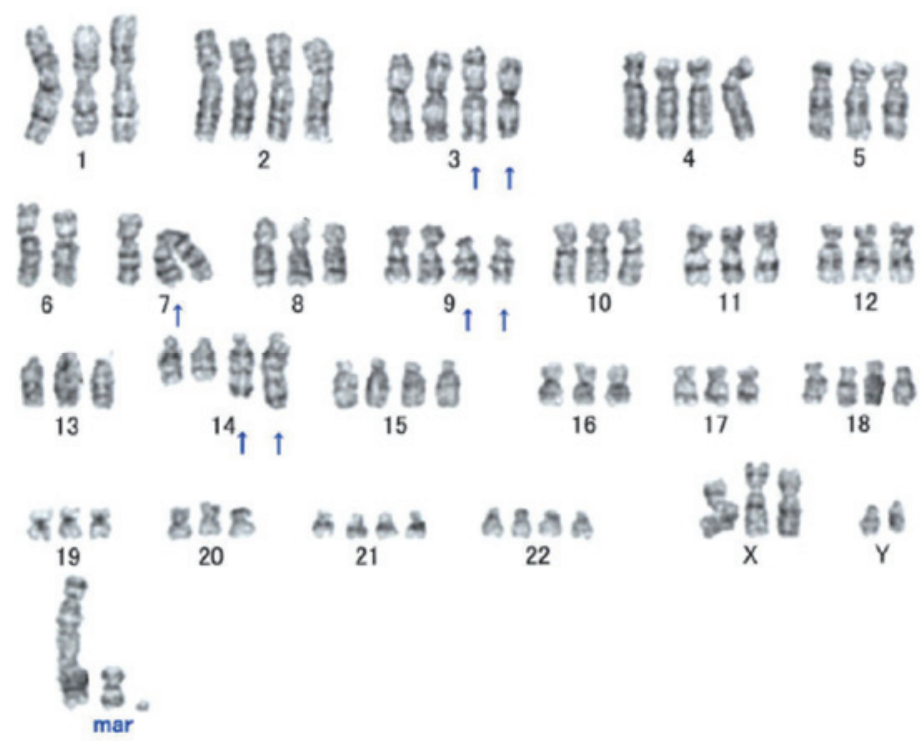

B

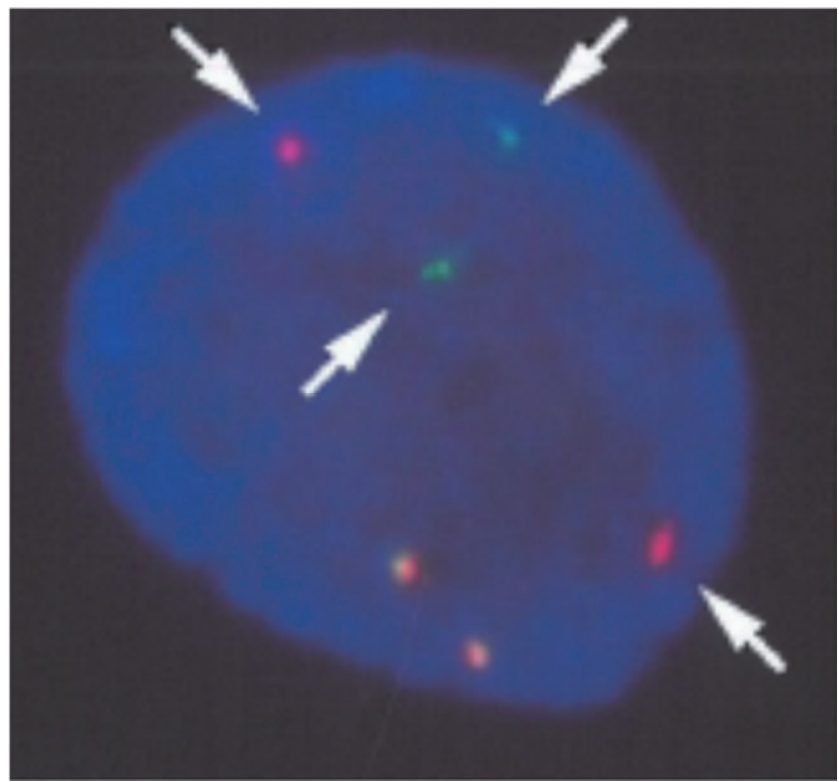

Fig. 3. (A) G-banded karyotype from the pleural effusion, revealing $82<3 \mathrm{n}>$, XXY, $+\mathrm{Y},+2,+\operatorname{der}(3)$ ?t $(3 ; 14)(\mathrm{q} 27 ; \mathrm{q} 32) \mathrm{x} 2, ? \mathrm{t}(3 ; 9 ; 14)$ $(\mathrm{q} 27 ; \mathrm{p} 13 ; \mathrm{q} 32),-6,-7, \operatorname{add}(7)(\mathrm{p} 22),+\operatorname{der}(9) ? \mathrm{t}(3 ; 9)(\mathrm{q} 27 ; \mathrm{p} 13),+13,+\operatorname{add}(14)(\mathrm{q} 32),+18,+19,+21,+5$ mar. $(\boldsymbol{B})$ Fluorescence in situ hybridization analysis for $B C L 6$ of pleural effusion. $B C L 6=$ yellow signal, 5 ' $B C L 6=$ red signal and 3 ' $B C L 6=$ green signal 
carcinoma, resulted in early interruption of the study after three of the eight treated patients developed significant ascites and/or pleural effusion, likely resulting from a marked reduction in interstitial fluid pressure. ${ }^{22}$ The negligible effects of imatinib on PDGFRB may explain the low incidence of this event with this agent. ${ }^{23-25}$ Another possible explanation is that tyrosine kinases responsible for capillary integrity may be overexpressed in the pulmonary vasculature and/or pleural epithelium. Src regulates focal adhesion and adherens junctions, two subcellular matrix attachment structures key in regulating cell adhesion, ${ }^{26,27}$ which may play a role in the stability of the pleural epithelium and pleural space homeostasis. The vascular permeability activity mediated by vascular endothelial growth factor is directly dependent on the Src-related kinases Yes and Src, which are widely expressed in lung tissue ${ }^{28,29}$ and inhibited by dasatinib. ${ }^{17,18,30}$ However, other dual Src and abl inhibitors, such as bosutinib, have not been associated with pleural effusion ${ }^{31}$ and bosutinib does not inhibit PDGFRB. ${ }^{32}$ Although there are some reports on the mechanism of TKI-related pleural effusion, PDGFRB rearrangement is usually associated with myeloid tumors, as typified by chronic eosinophilic leukemia, and the relationship with TKI-related pleural effusion and the onset mechanism of PEL-like lymphoma are unclear. Elucidation of their relevance is a future task.

To the best of our knowledge, this is the first report of treatment of PEL-like lymphoma following TKI treatment for CML. Accumulation of cases and further analysis are needed.

\section{CONFLICT OF INTEREST}

The authors have no conflicts of interest or funding to disclose.

\section{REFERENCES}

1 Campo E, Swerdlow SH, Harris NL, et al:: The 2008 WHO classification of lymphoid neoplasms and beyond: evolving concepts and practical applications. Blood 117: 5019-5032, 2011

2 Geary CG: The story of chronic myeloid leukaemia. Br J Haemtol 110: 2-11, 2000

3 Hoffmann VS, Baccarani M, Hasford J, et al.: Treatment and outcome of 2904 CML patients from the EUTOS populationbased registry. Leukemia 31: 593-601, 2017

4 Quintás-Cardama A, Kantarjian H, O'brien S, et al.: Pleural effusion in patients with chronic myelogenous leukemia treated with dasatinib after imatinib failure. J Clin Oncol 25: 39083914, 2007

5 Kantarjian H, Shah NP, Hochhaus A, et al.: Dasatinib versus imatinib in newly diagnosed chronic-phase chronic myeloid leukemia. N Engl J Med 362: 2260-2270, 2010

6 Saglio G, Kim DW, Issaragrisil S, et al.: Nilotinib versus imatinib for newly diagnosed chronic myeloid leukemia. N Engl J Med 362: 2251-2259, 2010

7 Cortes JE, Kim DW, Kantarjian HM, et al.: Bosutinib versus imatinib in newly diagnosed chronic-phase chronic myeloid leukemia results from the BELA trial. J Clin Oncol 30: 3486-3492, 2012

8 Carbone A, Gloghini A: KSHV/HHV8-associated lymphomas. Br J Haematol 140: 13-24, 2008

9 Kobayashi Y, Kamitsuji Y, Kuroda J, et al.: Comparison of human herpes virus 8 related primary effusion lymphoma with human herpes virus 8 unrelated primary effusion lymphomalike lymphoma on the basis of HIV: report of 2 cases and review of 212 cases in the literature. Acta Haematol 117;132144, 2007

10 Carbone A, Gloghini A: PEL and HHV8-unrelated effusion lymphomas classification and diagnosis. Cancer 114:225-227, 2008

11 Takahashi N, Miura I, Saitoh K, Miura AB: Lineage involvement of stem cells bearing the philadelphia chromosome in chronic myeloid leukemia in the chronic phase as shown by a combination of fluorescence in situ hybridization. Blood 92: 4758-4763, 1998

12 Ichinohasama R, Miura I, Takahashi N, et al.: Ph-negative nonHodgkin's lymphoma occurring in chronic phase of Ph-positive chronic myelogenous leukemia is defined as a genetically different neoplasm from extramedullary localized blast crisis: report of two cases and review of the literature. Leukemia 14(1): 169-182, 2000

13 Hochhaus A, Larson RA, Guilhot F, et al.: Long-Term Outcomes of Imatinib Treatment for Chronic Myeloid Leukemia. N Engl J Med 376(10): 917-927, 2017

14 Cortes JE, Saglio G, Kantarjian HM, et al.: Final 5-Year Study Results of DASISION: The Dasatinib Versus Imatinib Study in Treatment-Naïve Chronic Myeloid Leukemia Patients Trial. J Clin Oncol 34(20): 2333-2340. 2016

15 Kawamata T, Lu J, Sato T, et al.: Imatinib mesylate directly impairs class switch recombination through down-regulation of AID: its potential efficacy as an AID suppressor. Blood 119(13): 3123-3127. 2012

16 Quintás-Cardama A, Kantarjian H, O’brien S, et al.: Pleural effusion in patients with chronic myelogenous leukemia treated with dasatinib after imatinib failure. J Clin Oncol 25: 39083914, 2007

17 Lombardo LJ, Lee FY, Chen P, et al.: Discovery of N-(2-chloro6-methyl-phenyl)-2-(6-(4-(2-hydroxyethyl)-piperazin-1-yl)-2methylpyrimidin-4-ylamino)thiazole-5-carboxamide (BMS354825), a dual Src/Abl kinase inhibitor with potent antitumor activity in preclinical assays. J Med Chem 47: 6658-6661, 2004

18 Lee F, Lombardo, L, Camuso, A, et al.: BMS-354825 potently inhibits multiple selected oncogenic tyrosine kinases and possesses broad spectrum anti-tumor activities in vitro and in vivo. Proc Am Assoc Cancer Res 46: 159, 2005

19 Bergers G, Song S, Meyer-Morse N, et al.: Benefits of targeting both pericytes and endothelial cells in the tumor vasculature with kinase inhibitors. J Clin Invest 111: 1287-1295, 2003

20 Hellstrom M, Kalen M, Lindahl P, et al: Role of PDGF-B and PDGFR-beta in recruitment of vascular smooth muscle cells and pericytes during embryonic blood vessel formation in the mouse. Development 126: 3047-3055, 1999

21 Lindahl P, Johansson BR, Leveen P, et al.: Pericyte loss and 
microaneurysm formation in PDGF-B-deficient mice. Science 277:242-245, 1997

22 Jayson GC, Parker GJ, Mullamitha S, et al.: Blockade of platelet-derived growth factor receptor beta by CDP860, a humanized, PEGylated di-Fab', leads to fluid accumulation and is associated with increased tumor vascularized volume. J Clin Oncol 23: 973-981, 2005

23 Goldsby R, Pulsipher M, Adams R, et al.: Unexpected pleural effusions in 3 pediatric patients treated with STI-571. J Pediatr Hematol Oncol 24: 694-695, 2002

24 Park YH, Park HJ, Kim BS, et al: BNP as a marker of the heart failure in the treatment of imatinib mesylate. Cancer Lett 243:16-22, 2005

25 Breccia M, D'Elia GM, D'Andrea M, et al.: Pleural-pericardic effusion as uncommon complication in CML patients treated with imatinib. Eur J Haematol 74: 89-90, 2005

26 Carragher NO, Westhoff MA, Fincham VJ, et al: : A novel role for FAK as a protease-targeting adaptor protein: Regulation by p42 ERK and Src. Curr Biol 13: 1442-1450, 2003

27 Carragher NO, Frame MC: Calpain: A role in cell transformation and migration. Int J Biochem Cell Biol 34: 1539-1543, 2002
28 Luton F, Verges M, Vaerman JP, et al.: The SRC family protein tyrosine kinase p62yes controls polymeric IgA transcytosis in vivo. Mol Cell 4: 627-632, 1999

29 Thomas SM, Brugge JS: Cellular functions regulated by Src family kinases. Annu Rev Cell Dev Biol 13: 513-609, 1999

30 Abram CL, Courtneidge SA: Src family tyrosine kinases and growth factor signaling. Exp Cell Res 254: 1-13, 2000

31 Cortes J, Kantarjian, H, Baccarani, M, et al.: A phase 1/2 study of SKI-606, a dual inhibitor of Src and Abl kinases, in adult patients with Philadelphia chromosome positive $(\mathrm{Ph}+)$ chronic myelogenous leukemia (CML) or acute lymphocytic leukemia (ALL) relapsed, refractory or intolerant of imatinib. Blood 108, 2006 (abstr 168)

32 Puttini $\mathrm{M}$, Coluccia AM, Boschelli $\mathrm{F}$, et al.: In vitro and in vivo activity of SKI-606, a novel Src-Abl inhibitor, against imatinibresistant Bcr-Abl+ neoplastic cells. Cancer Res 66: 1131411322, 2006 